\title{
GENERALIDADES DEL PARQUE NACIONAL VOLCÁN IRAZÚ
}

\author{
GENERAL ASPECTS OF IRAZÚ VOLCANO NATIONAL PARK
}

\author{
Alejandra Varela \\ Encargada Programa Turismo Sostenible, Parque Nacional Volcán Irazú, \\ Área de Conservación Cordillera Volcánica Central (ACCVC)-SINAC, \\ Ministerio de Ambiente, Energía y Telecomunicaciones, \\ Apdo. 10104-1000, San José, Costa Rica. \\ alejandra.varela@sinac.go.cr \\ (Recibido: 17/9/2012; aceptado: 11/6/2013)
}

\begin{abstract}
The Irazú Volcano National Park was created on July $30^{\text {th }}, 1955$. Its name probably comes from a native word "Istarú", which means Thunder and Earthquake Mountain. This National Park has two scenic areas: The Craters area and Prusia area. Visitors can enjoy trails and do recreational activities. Irazú summit (3432 m a.s.1.) offers a variety of attractions: volcanic landsforms (craters, crater lakes, cones), and elsewhere it is possible to find species common of subalpine paramo elevations.

Keywords: Irazú Volcano National Park, Costa Rica

RESUMEN: El Parque Nacional Volcán Irazú fue creado en julio de 1955. Su nombre proviene de la palabra indígena "Istarú" que significa Cerro del Temblor y del Trueno. Este Parque Nacional posee dos sectores: El Sector Cráteres y el Sector Prusia. Los visitantes podrán disfrutar de senderos y hacer actividades recreativas. La cumbre del Irazú (3432 m s.n.m.) ofrece una variedad de atracciones: geoformas volcánicas, lagunas cratéricas y conos y donde sea es posible encontrar especies típicas de páramo subalpino.

Palabras clave: Parque Nacional Volcán Irazú, Costa Rica
\end{abstract}




\section{ANTECEDENTES}

Las Áreas Silvestres Protegidas (ASP) de Costa Rica son espacios fundamentales para la conservación de los ecosistemas naturales y de la biodiversidad. Para el año 1955, mediante la Ley No.1917, se declararon como parques nacionales las zonas comprendidas dentro de un radio de dos kilómetros alrededor de los cráteres de los principales volcanes del país. En este período, se establecen los Parques Nacionales Volcán Irazú y Volcán Turrialba (Bermúdez \& Conejo, 2008).

\section{Características del Parque Nacional Volcán Irazú}

El Parque Nacional Volcán Irazú (PNVI) se encuentra ubicado en la provincia de Cartago y pertenece al Área de Conservación Cordillera Volcánica Central (ACCVC), la cual recibió en 1988 la denominación de Reserva de la Biosfera por la UNESCO.

El volcán posiblemente recibe su nombre del vocablo indígena "Iztarú", que a través de los años se modificó a Irazú, y significa “cerro del temblor y del trueno" (Fig. 1). Dentro de sus principales rasgos destacan sus cráteres y la altura (3 432 m s.n.m.) siendo así el volcán más alto del país (Alvarado, 2009).

El Parque Nacional Volcán Irazú se amplía en 1997 mediante el Decreto Ejecutivo $\mathrm{N}^{\circ}$ 26945-MINAE, cuando se anexa el área de Prusia (área recuperada de la devastación ocasionada por las erupciones del Irazú en 1963-65). El decreto indicado se hizo efectivo cuando se publicó en la Gaceta Ofícial № 98, el 22 de mayo de 1998. La extensión del territorio declarado como parque nacional actualmente es de 2000,36 hectáreas (Bermúdez \& Conejo, 2008). Cabe destacar, que el manejo que se le da al parque nacional, se desprende de un documento llamado
Plan General de Manejo, y anualmente se debe cumplir con lo programado en él, incluyéndolo dentro del Plan Anual de Trabajo (PAT). Además, se complementa con lo que sugiere el Plan de Turismo Sostenible y el Monitoreo de la Gestión.

El personal a cargo realiza giras de control y protección, tanto dentro del ASP como en las comunidades aledañas, en las cuales también se realiza educación ambiental, con el fin de concientizar a la población sobre la importancia de proteger los recursos naturales. A continuación, se mencionaran los aspectos más relevantes del parque.

\section{Dos sectores}

Los turistas tienen la posibilidad de visitar los dos sectores del parque. El Sector Cráteres, donde es posible observar cinco cráteres, caminar por una antigua "caldera" volcánica y observar desde el mirador del punto más alto el imponente volcán Turrialba, el cual deja ver su columna de gas en días soleados.

Y el sector Prusia, una zona boscosa, ideal para quienes gustan de caminar por senderos, donde es posible la observación de aves y hongos, dos de sus atractivos más llamativos.

Ambos lugares, a pesar de pertenecer a un mismo parque nacional, tienen características diferentes; esta es otra de las maravillas que presenta este sitio.

\section{Sector Cráteres}

Como consecuencia de la actividad volcánica y la gran altura a la que se encuentra la cima del Irazú, se propicia el desarrollo de vegetación del tipo páramo subalpino. Dentro de 


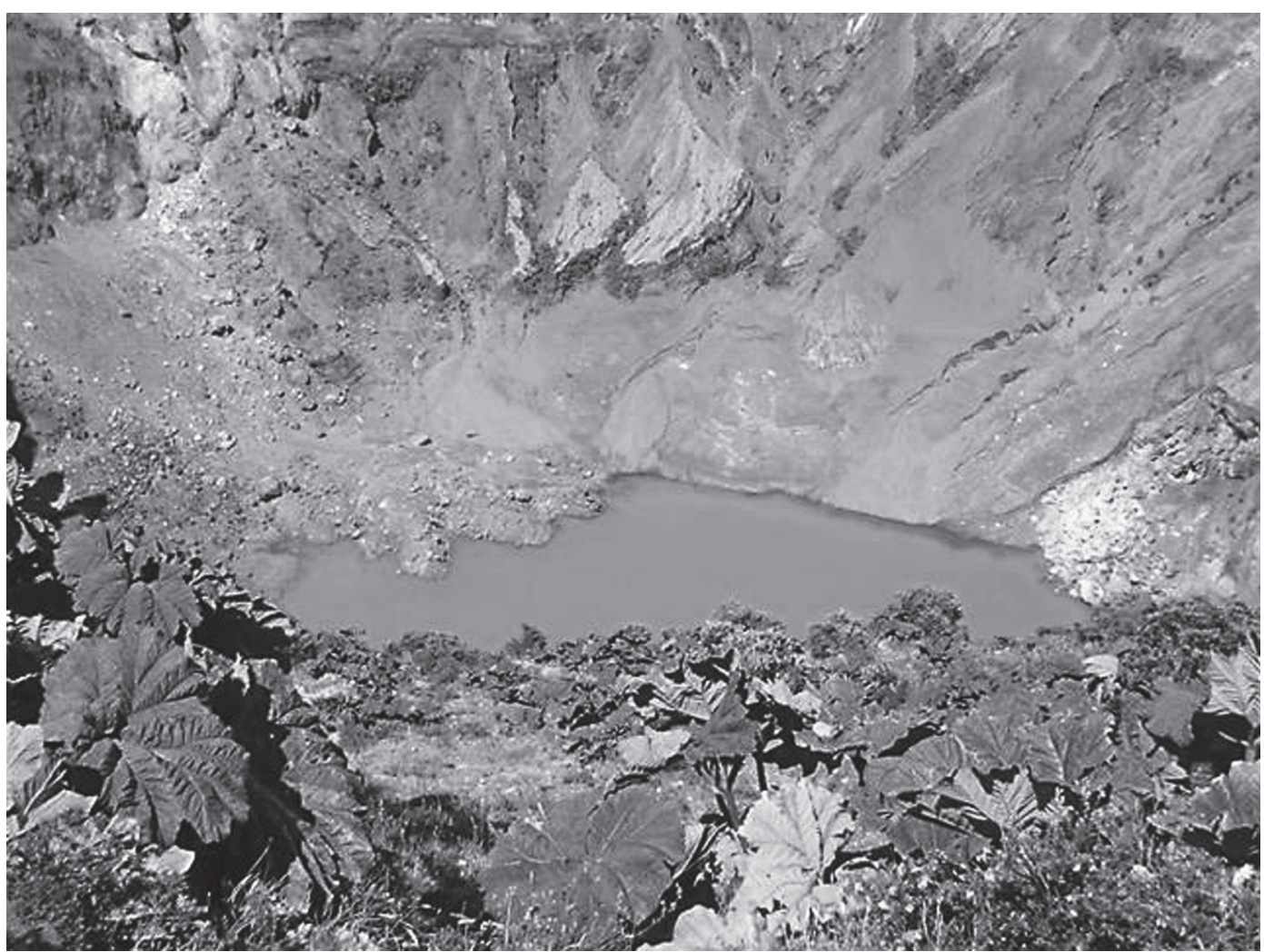

Fig. 1: Cráter Principal del Volcán Irazú (Fotografía: Yuliana Rivera B.).

las especies de plantas más representativas se encuentran la castilleja (Castilleja irazuensis) que es endémica del Irazú (Fig. 2) (Morales \& Bermúdez, 2002), aunque a través de los años se ha trasladado también hacia el Parque Nacional Volcán Turrialba. También se cuenta con la sombrilla de pobre (Gunnera insignis) llamativa por sus hojas grandes, rugosas y de forma muy similar a una sombrilla, de ahí su nombre.

A la fecha no se cuenta registros recientes de la totalidad de especies animales de la zona. No obstante, el proyecto "Monitoreo de
Mamíferos" ha identificado algunos ejemplares. Asimismo, y pese a su escasez, la fauna del lugar es bastante variada, encontrándose desde pequeños mamíferos hasta diversos tipos de aves. De las especies animales existentes en el Irazú destacan los puercoespines (Coendou mexicanus), coyotes (Canis latrans), conejos (Silvilagus sp.), armadillos (Dasypus novemcinctus), y la zorra gris (Urocyon cinereoargenteus). Ver Fig. 3.

La población de aves del Parque, abarca una amplia gama de ejemplares, dentro de la cual que se pueden encontrar el yigüirro escarchero 


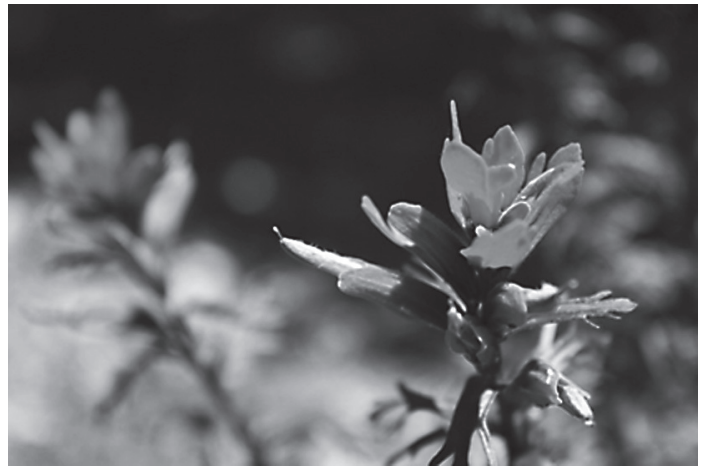

Fig. 2: Flor de la Castilleja irazuensis (Fotografía: Juan Carlos Sibaja).

(Turdus nigrescens), tángara de monte cejiblanca (Chlorospingus pileatus) y junco vulcanero (Junco volcanis).

\section{Sector Prusia}

En el Sector Prusia, la vegetación natural fue destruida por las erupciones de 1963-65. Para tratar de proteger los suelos y evitar nuevas avalanchas sobre la ciudad de Cartago, se realizó un proyecto de reforestación intensiva utilizando tanto especies exóticas como nativas. Las especies introducidas fueron: ciprés, pino y eucalipto; mientras que las especies nativas utilizadas fueron: roble (Quercus sp.), jaúl (Alnus sp.) y salvia (Buddleja sp.) (Morales \& Bermúdez, 2002). En este sector es posible caminar por hermosos senderos, llenos de exuberante vegetación. Una de sus bellezas más sobresaliente es la posibilidad de observar hongos de diversas especies, uno de los más llamativos por su colorido es el hongo Amanita muscaria (Fig. 4).

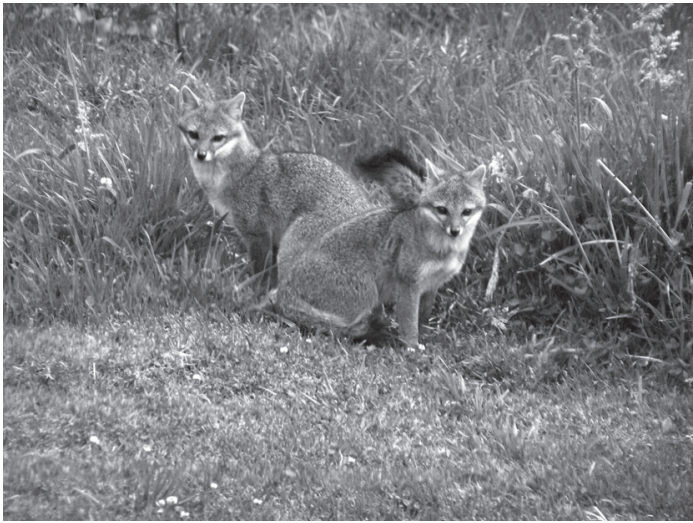

Fig. 3: Zorras grises (Urocyon cinereoargenteus) Sector Prusia (Fotografía: Horacio Herrera S.).

El clima es más cálido que en el sector cráteres, por lo que es ideal para relajarse y compartir con la naturaleza. Es un lugar tranquilo donde se escucha el cantar de las aves.

\section{CLIMA}

Durante los días más fríos, en la cima del volcán Irazú, la temperatura es ligeramente inferior al punto de congelación. La temperatura media anual varía de $5^{\circ} \mathrm{C}$ a $9^{\circ} \mathrm{C}$.; la temperatura máxima promedio es de $17^{\circ} \mathrm{C}$ y la mínima de $-3^{\circ} \mathrm{C}$, lo que permite en ciertos meses del año apreciar escarcha en la cima del volcán y raramente nieve.

La estación meteorológica Sanatorio Durán, en las cercanías del Sector Prusia, presenta un promedio de precipitación de 1527,9 mm anual. La estación seca se limita a los meses de febrero y marzo. La temperatura media anual en este sector es de $14^{\circ} \mathrm{C}$. (Bermúdez \& Conejo, 2008) 


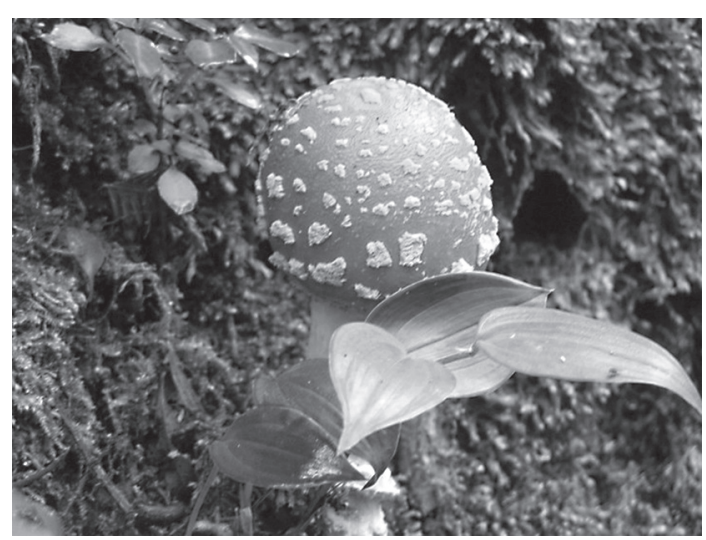

Fig. 4: Hongo tipo Amanita muscaria, Sector Prusia (Fotografía: Horacio Herrera S.).

\section{GEOLOGÍA}

El volcán Irazú se clasifica como un escudo andesítico activo, lo que significa que está formado por corrientes extensas de coladas de lava y rocas piroclásticas, dependientes suaves a moderadas con una forma subcónica irregular.

La zona intra-cratérica se encuentra formada por tres estructuras principales; el cráter activo (Fig. 1) hacia el noroeste con aproximadamente $1000 \mathrm{~m}$ de diámetro, una profundidad aproximada de $300 \mathrm{~m}$ con un lago de color y características variables; el cráter Diego de la Haya que se encuentra al norte de la "caldera" con unos $80 \mathrm{~m}$ de profundidad y de forma alargada hacia el este; y finalmente al sur una larga estructura semi-plana que corresponde a los restos de una terraza volcánica antigua conocida con el nombre de Playa Hermosa. Fuera de la gran caldera también se nota al este un cono piroclástico, bien preservado de unos 80 $\mathrm{m}$ de altura (Alvarado, 2009)

\section{Visitación}

El Parque Nacional Volcán Irazú es el tercer parque más visitado en Costa Rica, superado solo por los parques nacionales Manuel Antonio y Volcán Poás (SEMEC, 2012). Diariamente el Irazú es visitado por nacionales y extranjeros, atraídos por los paisajes hermosos y la posibilidad de observar una laguna intra-cratérica color verde (Fig. 1). Muchos de los nacionales, disfrutan en horas de la mañana la posibilidad de observar "escarcha", fenómeno considerado por algunos visitantes una rareza en un país tropical (Fig. 5). Dentro de las nacionalidades extranjeras que más destacan por su visita se encuentran: franceses, holandeses y alemanes, los cuales disfrutan del clima frío de la cima y el avistamiento de aves.

Uno de sus atractivos es la posibilidad de transitar por dos sectores diferentes, permitiendo al visitante disfrutar de dos tipos de ambiente:

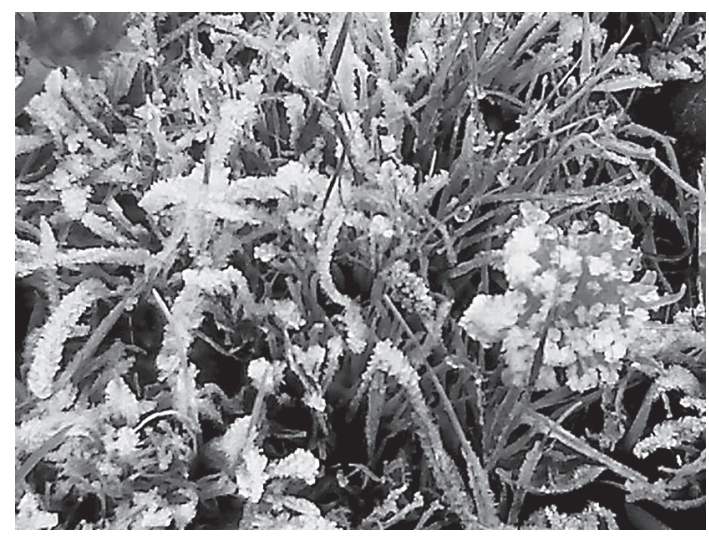

Fig. 5: Vegetación cubierta por hielo debido a las bajas temperaturas (Fotografía Alejandra Varela S.). 


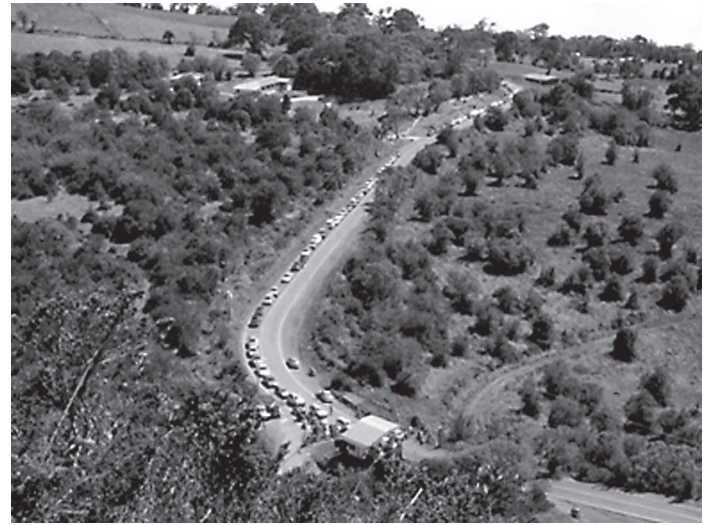

Fig. 6: Vista de la entrada al Parque Nacional Volcán Irazú y su gran afluencia de vehículos (Fotografía Juan Carlos S.).

Prusia y Cráteres. Presenta la peculiaridad de tener acceso a los principales cráteres en cualquier tipo de vehículo, siendo esta característica poco común globalmente.

\section{Actividades permitidas}

El espacio que se brinda para la recreación debe servir fundamentalmente para crear conciencia sobre la importancia de proteger los recursos naturales. Es por ello, que se permite a los visitantes la entrada en un horario controlado, es decir, de lunes a domingo de 8:00 am a 3:30 pm, pero debe tomarse en cuenta que los domingos, especialmente durante la época seca, son de alta afluencia de visitantes, estos días se puede durar hasta dos horas para poder ingresar, por lo que deben tener paciencia (Figs. 6 y 7).

Se puede traer alimentos, aunque se cuenta con una cafetería y tienda de artesanías.

Se deben conservar los tiquetes que se entregan en la caseta de entrada, los que le servirán para ingresar a los dos sectores del parque nacional. Los teléfonos para información son 2200-5025 en Sector Cráteres y 2200-4422 en Sector Prusia.

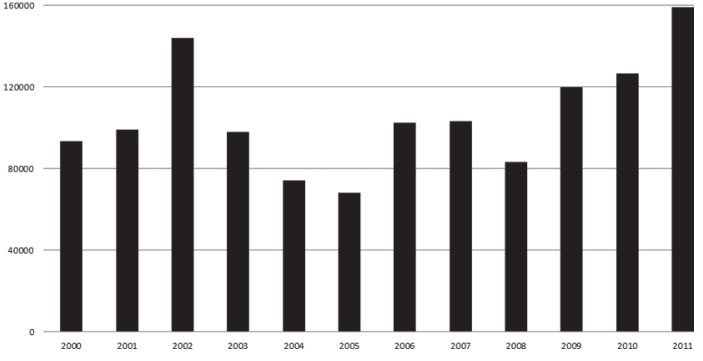

Fig. 7: Estadísticas de visitación del Parque Nacional Volcán Irazú (según consulta a SEMEC, 2012).

\section{Actividades no permitidas}

Se les solicita no ingresar al área de cráteres con alimentos y no alimentar a la fauna. Ya que se tiene la posibilidad de admirar animales silvestres, no se admite el ingreso de animales domésticos.

Los senderos han sido construidos para el disfrute y seguridad de los visitantes. Por ello manténgase dentro de los límites establecidos. Tampoco se permite la ingesta de bebidas alcohólicas ni el fumado.

El parque es de todos los costarricenses, permita que otros lo conozcan, no extraiga plantas ni rocas.

\section{AGRADECIMIENTOS}

Gracias a todos los funcionarios del Área de Conservación Cordillera Volcánica Central (ACCVC) por los datos proporcionados para la realización de este trabajo. Especialmente a los compañeros: Alejandro Moya, Paola Rodríguez, Daniel Núñez, Horacio Herrera, Olga Durán, Damaris Garita y Mauricio Arias. Al Dr. Guillermo Alvarado Induni, del Instituto Costarricense de Electricidad y la Universidad de Costa Rica, por la paciencia y la ayuda brindada. A los estudiantes del programa de voluntariado 
de la Universidad de Costa Rica (UCR), Aileen Orozco, Karol Gómez y Juan Carlos Sibaja. Y a mi esposo, Rubén Valverde, por toda la colaboración brindada.

\section{REFERENCIAS}

ALVARADO, G.E., 2009: Los volcanes de Costa Rica: Geología, historia, riqueza natural y su gente.335 págs. EUNED (3 $3^{\text {era }}$ edición); San José.

BERMÚDEZ, F. \& CONEJO, R., 2008: Plan General de Manejo del Parque Nacional
Volcán Irazú.120 págs.- Onca Natural, San José, Costa Rica. http: www.acvc.org [Consulta: 17 de setiembre de 2012].

MORALES, R.\& BERMÚDEZ, F.,2002. Plan de Manejo del PNVI. ACCVC. Cartago, CR.- http: //www.accvc.org [Consulta: 16 de noviembre 2011].

SEMEC, 2012: Datos de visitación, Informes SEMEC 2000-2011.- Área de Conservación Cordillera Volcánica Central (ACCVC). Heredia.- http: www.acvc.org [Consulta: 22 de mayo de 2012]. 
